\title{
The Power of a Propositional Constant
}

\author{
Robert Goldblatt and Tomasz Kowalski
}

2 November 2012

\begin{abstract}
Monomodal logic has exactly two maximally normal logics, which are also the only quasi-normal logics that are Post complete, and they are complete for validity in Kripke frames. Here we show that addition of a propositional constant to monomodal logic allows the construction of continuum many maximally normal logics that are not valid in any Kripke frame, or even in any complete modal algebra. We also construct continuum many quasi-normal Post complete logics that are not normal.

The set of extensions of S4.3 is radically altered by the addition of a constant: we use it to construct continuum many such normal extensions of S4.3, and continuum many non-normal ones, none of which have the finite model property.

But for logics with weakly transitive frames there are only eight maximally normal ones, of which five extend K4 and three extend S4.
\end{abstract}

\section{Introduction}

This is a study of some properties of the set of propositional modal logics in a language that has a one-place modality $\square$, with dual $\diamond$, and a constant that is interpreted as a fixed proposition (set of worlds) in a Kripke frame. There are a number of natural examples of such logics, suggesting that a general meta-study would be worthwhile. Here are some such examples:

Example 1.1. Anderson [2] constructed a deontic logic by starting with a normal alethic logic, in which $\square$ is interpreted as 'necessarily', adding a constant $\mathscr{P}$, and defining 'it is obligatory that $p$ ' as $\square(\neg p \rightarrow \mathscr{P} \wedge \triangleright \neg \mathscr{P})$. The idea is that $\mathscr{P}$ describes 'some "bad" state-of-affairs', and that 'to say that $p$ is obligatory is to say that failure of $p$ leads to [i.e. necessarily implies] a state-of-affairs $\mathscr{P}$ which is "bad", but avoidable $(\diamond \neg \mathscr{P})$ '.

Prior [21] simplified this analysis by introducing a propositional constant $\mathscr{S}$, which he called 'the sanction', with the same intended meaning as $\mathscr{P} \wedge \diamond \neg \mathscr{P}$. Defining 'it is obligatory that $p$ ' as $\square(\neg p \rightarrow \mathscr{S})$, he observed that if the alethic logic includes the system KT, then only the axiom $\diamond \neg \mathscr{S}$ ('it is possible to escape the sanction') is needed to derive the standard deontic principles about obligation. ${ }^{1}$

\footnotetext{
${ }^{1}$ Further philosphical discussion of this topic is given in [19].
} 
Example 1.2. The propositional dynamic logic of programs [12] can be extended by assigning to each program $\alpha$ a constant proposition repeat $\alpha$ which asserts that $\alpha$ can be repeatedly executed infinitely many times, and which is true at a given state when there is an infinite sequence of states emanating from the given one, with each being obtained from the previous one by an $\alpha$ action. The resulting logic was shown to be decidable by Streett [25], using automata-theoretic methods.

This type of propositional constant was investigated by Goldblatt [10], studying modal algebras that have a distinguished element $\lambda$ that is a greatest fixed point of the operator interpreting the modality $\diamond$. These algebras were shown to form a quasi-variety with a decidable equational theory, and to be generated by the algebras of subsets of (finite) Kripke frames $(W, R)$ in which $\lambda$ denotes the set of points $x$ for which there exists an infinite sequence $x R x_{1} R x_{2} R \cdots \cdots$.

Example 1.3. The notion of a monadic bounded algebra (MBA) was introduced by Akishev and Goldblatt [1] as a Boolean algebra with a unary operator $\exists$ and an individual constant $E$, satisfying certain equations. This generalizes Halmos's notion of a monadic algebra [11], and is intended to capture properties of the existential quantifier in free logic, i.e. logic without existence assumptions, in which $E$ represents an existence assertion. Alternatively an MBA may be viewed as capturing properties of the bounded quantifier 'for some member of E'.

A classification was given in [1] of all the equationally definable classes of MBA's. These correspond exactly to normal modal logics that extend the logic $\mathrm{K} 45^{2}$ in a language with a propositional constant $E$, and contain the axioms $p \wedge E \rightarrow \diamond p$ and $\diamond p \rightarrow \diamond(p \wedge E)$. There are denumerably many such logics; all are finitely axiomatizable and have the finite model property.

Example 1.4. A modal logic is regular for $\square$ if it is closed under the derivation of $\square A \wedge \square B \rightarrow \square C$ from $A \wedge B \rightarrow C$. A $\square$-regular logic is normal if it contains $\square \top$, where $T$ is a constant universally true formula. Given a $\square$-normal logic in a language that has an additional constant $\mathscr{N}$, we introduce a new modality $\boxplus$ by defining $\boxplus A$ to be the formula $\mathscr{N} \wedge \square A$. Then the logic is $\boxplus$-regular, but need not be $\boxplus$-normal because $\boxplus \top$ is $\mathscr{N} \wedge \square T$, so by $\square$-normality we get only that

$$
\boxplus \top \leftrightarrow \mathscr{N}
$$

is derivable. To see if $\square$ can be recovered from $\boxplus$, we use $\boxplus$ to define another modality $\square^{\prime}$ by putting $\square^{\prime} A=\mathscr{N} \rightarrow \boxplus A$. The logic is then $\square^{\prime}$-normal, with $\square^{\prime} \top$ being the derivable $\mathscr{N} \rightarrow \boxplus \top$. In general we have that

$$
\square^{\prime} A \leftrightarrow(\mathscr{N} \rightarrow \square A)
$$

is derivable. Now if the formula $\mathscr{N} \vee \square \perp$ is derivable, then since $\square \perp \rightarrow \square A$ is derivable by $\square$-regularity, we can derive $(\mathscr{N} \rightarrow \square A) \rightarrow \square A$, and from that,

\footnotetext{
${ }^{2}$ With proper axioms $\square p \rightarrow \square \square p$ and $\diamond \square p \rightarrow \square p$. Also known as KE4.
} 
$(\mathscr{N} \rightarrow \square A) \leftrightarrow \square A$. Hence by (1.2), $\square^{\prime} A \leftrightarrow \square A$ is derivable, and in this sense $\square$ is recovered from $\boxplus$.

In the converse direction, starting with a language having only a modality $\boxplus$, (1.1) and the definition of $\square^{\prime}$ suggest that $\square$ and $\mathscr{N}$ could be introduced by defining $\square A$ to be $\mathscr{N} \rightarrow \boxplus A$, where $\mathscr{N}$ is defined to be $\boxplus \top$. A logic that is $\boxplus$ regular will then be $\square$-normal for $\square$ thus defined, with $\square T$ being the tautology $\boxplus \top \rightarrow \boxplus \top$.

$\boxplus$ can now be recovered from $\square$ and $\mathscr{N}$, because if we put $\boxplus^{\prime} A=\mathscr{N} \wedge \square A$, then $\boxplus^{\prime} A=\boxplus \top \wedge(\boxplus \top \rightarrow \boxplus A)$, which is equivalent to $\boxplus A$ as $\boxplus A \rightarrow \boxplus \top$ is derivable by $\boxplus$-regularity. Thus $\boxplus^{\prime} A \leftrightarrow \boxplus A$ is derivable. Moreover $\mathscr{N} \vee \square \perp$ is derivable, since it is the tautology $\boxplus \top \vee(\boxplus \top \rightarrow \boxplus \perp)$.

These constructions lead to a bijective correspondence between regular logics in a language with $\boxplus$ and normal logics, in a language with $\square$ and a propositional constant $\mathscr{N}$, that contain $\mathscr{N} \vee \square \perp$. The relationships are reflected in Kripke's semantics for non-normal logics [16], which uses models based on frames of the form ( $W, R, N)$ with $N \subseteq W$. Members of $N$ are thought of as 'normal' worlds. Such a model interprets a normal modality $\square$ by making $\square A$ true at a point $x$ of $W$ iff $A$ is true throughout $\{y \in W: x R y\}$. It interprets a regular modality $\boxplus$ by making $\boxplus A$ true at $x$ iff $x \in N$ and $A$ is true throughout $\{y \in W: x R y\}$. If $\mathscr{N}$ is interpreted as the set $N$, it follows that the model verifies $\boxplus A \leftrightarrow \mathscr{N} \wedge \square A$ and $\boxplus \top \leftrightarrow \mathscr{N}$. Also $\square A \leftrightarrow(\mathscr{N} \rightarrow \boxplus A)$ is verified if the model verifies $\mathscr{N} \vee \square \perp$.

We turn now from examples to generalities. A great deal is known about the metatheory of logics that are monomodal (have $\square$ but no constant) or are bimodal (have two one-place modalities), and there are considerable differences in behaviour between the two kinds. For instance, the first discovered example [26] of a normal logic that is incomplete for Kripke semantics was a bimodal one, in fact a linear tense logic, with the property that it is not valid in any Kripke frame at all. This cannot happen for monomodal logics, due to results of Makinson [18] implying that any consistent normal monomodal logic is valid in a one-element frame. There are two such frames: the reflexive one determining the 'trivial' logic Triv with proper axiom $\square p \leftrightarrow p$, and the irreflexive one determining the 'verum' logic Ver with axiom $\square p$. So any consistent normal logic is a sublogic either of Triv or Ver, and these two are the only maximally normal monomodal logics. They are also the only monomodal logics that both include the smallest normal logic K (i.e. are quasi-normal) and are Post complete in the sense of having no consistent extensions at all.

By contrast, Williamson [28] showed that there are continuum many $\left(2^{\aleph_{0}}\right)$ bimodal logics that are maximally normal, each of which has infinitely many Post complete extensions. Kowalski [14] proved a similar result for tense logics. Recently, French [9] defined denumerably many $\left(\aleph_{0}\right)$ maximally normal logics in the language with a single modality and an additional constant $\kappa$, each being the logic determined by some Kripke frame. He asked if there are in fact nondenumerably many maximally normal logics in this language.

We give a positive answer to this question here. After developing some 
general theory in Section 2, we use the constant $\kappa$ to construct in Section 3 a continuum of maximally normal logics, all of which are 'anti-complete' for Kripke semantics, in that they are not valid in any Kripke frames. From these we then obtain continuum many quasi-normal Post complete logics that are not normal. All of these logics include the reflexivity axiom $\square p \rightarrow p$.

If instead we consider logics with the transitivity axiom $4: \diamond \diamond p \rightarrow \diamond p$, the situation is completely different. In Section 4 we show that are exactly five maximally normal extensions of K4 in the language with $\kappa$, three of which are the only maximally normal extensions of S4. Relaxing axiom 4 to the weaker $4^{w}: \diamond \diamond p \rightarrow p \vee \diamond p$ admits an additional three, giving altogether exactly eight maximally normal extensions of $\mathrm{K} 4^{w}$. Each is defined as the logic determined by some Kripke frame with either one or two elements. Four of them are Post complete (the ones determined by the singleton frames).

The final Section 5 examines logics extending S4.3, and shows that none of the 'tame' properties discovered by Bull [4], Fine [7] and Segerberg [24] for monomodal extensions of S4.3 survive in the presence of $\kappa$. We use the constant to construct continuum many normal extensions of S4.3, and continuum many non-normal ones, all of which lack the finite model property.

\section{$2 \quad$ Frames and Logics}

Fix a language whose atomic formulas consist of a set of propositional variables $p, q, \ldots$ and the propositional constants $\perp$ (falsity) and $\kappa$. Formulas $A, B, \ldots$ are constructed from these by the implication connective $\rightarrow$ and the one-place modality $\square$. The truth-functional connectives $\neg, \wedge, \vee, \leftrightarrow$ and the dual modality $\diamond$ are introduced in the usual way, and we write $\top$ for $\neg \perp$. Formulas $\square^{n} A$ are defined by induction on the natural number $n$ by putting $\square^{0} A=A$ and $\square^{n+1} A=\square\left(\square^{n} A\right)$. Formulas $\nabla^{n} A$ are defined likewise by iterating $\diamond$. A formula with no occurrence of $\kappa$ will be called monomodal, or $\kappa$-free. A formula with no variables will be called closed.

A substitution is a function $\sigma$ assigning to each variable $p$ a formula $\sigma p$. It is a closed substitution if $\sigma p$ is a closed formula for all $p$. Any substitution extends uniquely by induction on formation to a map $A \mapsto \sigma A$ on all formulas that has $\sigma \kappa=\kappa$ and commutes with the connectives. $\sigma A$ will be called a substitution instance of $A$.

A Kripke frame is a structure $\mathcal{F}=(W, R,|\kappa|)$, comprising a binary relation $R$ on a non-empty set $W$, and subset $|\kappa|$ of $W$ that will be the interpretation of the constant $\kappa$. A general frame $\mathcal{F}=(W, R,|\kappa|, P)$ has in addition a non-empty collection $P$ of subsets of $W$ that contains $|\kappa|$ and is closed under the Boolean set operations and under the operation $[R]$ that interprets $\square$, i.e.

$$
[R] X=\{x \in W: x R y \text { implies } y \in X\} .
$$

Then $P$ is closed under the operation $\langle R\rangle$ that interprets $\diamond$, having $\langle R\rangle X=$ $\{x \in W: \exists y \in X(x R y)\}$. 
The members of $P$ are the admissible propositions of $\mathcal{F}$. A Kripke frame $(W, R,|\kappa|)$ will be identified with the general frame $(W, R,|\kappa|, \wp W)$, where $\wp W$ is the powerset of $W$, in which all subsets of $W$ are admissible. We use the relations $R^{n}$ on $W$, where $R^{0}$ is the identity relation, and $R^{n+1}$ is the composition of $R$ with $R^{n}$.

A model $\mathcal{M}$ on a general frame is given by a function $|-|^{\mathcal{M}}$ that assigns to each variable $p$ a subset $|p|^{\mathcal{M}}$ of $W$. This is extended inductively to assign a truth set $|A|^{\mathcal{M}}$ to each formula $A$ by putting $|\perp|^{\mathcal{M}}=\emptyset,|\kappa|^{\mathcal{M}}=|\kappa|, \mid A \rightarrow$ $\left.B\right|^{\mathcal{M}}=\left(-|A|^{\mathcal{M}}\right) \cup|B|^{\mathcal{M}}$, and $|\square A|^{\mathcal{M}}=[R]|A|^{\mathcal{M}}$. If $x \in|A|^{\mathcal{M}}$ we may say that $A$ is true in $\mathcal{M}$ at $x$, and write $\mathcal{M}, x \models A$, or even $x \models A$ if the model is understood. $A$ is true in model $\mathcal{M}$, written $\mathcal{M} \models A$, if $|A|^{\mathcal{M}}=W$, i.e. if $A$ is true in $\mathcal{M}$ at every member of $W$.

Formula $A$ is valid in frame $\mathcal{F}$, written $\mathcal{F} \models A$, if it is true in every model on $\mathcal{F}$. We also say that $A$ is valid at $x$ in $\mathcal{F}$, written $\mathcal{F}, x \models A$, if it is true at $x$ in every model on $\mathcal{F}$. If $A$ is closed, then its truth at $x$ is model-independent, so that $A$ is valid at $x$ in $\mathcal{F}$ iff it is true at $x$ in at least one model on $\mathcal{F}$. Another way to capture this is to observe that each closed formula $A$ defines a subset $|A|^{\mathcal{F}}$ of $W$ inductively by putting $|\perp|^{\mathcal{F}}=\emptyset,|\kappa|^{\mathcal{F}}=|\kappa|,|A \rightarrow B|^{\mathcal{F}}=\left(-|A|^{\mathcal{F}}\right) \cup|B|^{\mathcal{F}}$, and $|\square A|^{\mathcal{F}}=[R]|A|^{\mathcal{F}}$. Then $|A|^{\mathcal{F}}=|A|^{\mathcal{M}}$ for every model $\mathcal{M}$ on $\mathcal{F}$, and $|A|^{\mathcal{F}}=\{x \in W: \mathcal{F}, x \models A\}$. We may write $|A|^{\mathcal{F}}$ just as $|A|$ if the frame is understood. $\mathcal{F}$ will be called a definable frame if each member of its set $P$ of admissible propositions is equal to $|A|$ for some closed formula $A .^{3}$

Lemma 2.1. If $\mathcal{F}$ is a definable frame, and $\mathcal{F} \not \forall A$, then $\mathcal{F} \forall \sigma A$ for some closed substitution $\sigma$.

Proof. If $\mathcal{F} \not \mid A$, then there is a model $\mathcal{M}$ on $\mathcal{F}$ with $|A|^{\mathcal{M}} \neq W$. If $\mathcal{F}$ is definable, then for each variable $p$ there is a closed formula $\sigma p$ with $|p|^{\mathcal{M}}=|\sigma p|$. This gives a closed substitution $\sigma$ for which a straightforward induction shows that $|B|^{\mathcal{M}}=|\sigma B|$ for all formulas $B$. Hence $|\sigma A| \neq W$, and so $\mathcal{F} \not \neq \sigma A$.

We will need the notion of a a function $f: W \rightarrow W^{\prime}$ being a bounded morphism from a frame $\mathcal{F}=(W, R,|\kappa|, P)$ to a frame $\mathcal{F}^{\prime}=\left(W^{\prime}, R^{\prime},|\kappa|^{\prime}, P^{\prime}\right)$. This means that

- $X \in P^{\prime}$ implies $f^{-1} X \in P$ (reflection of admissibility);

- $x R y$ implies $f x R^{\prime} f y$ (forth condition);

- $f x R^{\prime} z$ implies $\exists y(x R y \& f y=z)$ (back condition);

- $|\kappa|=f^{-1}|\kappa|^{\prime}$, i.e $x \in|\kappa|$ iff $f x \in|\kappa|^{\prime}$ ( $\kappa$-invariance).

Validity is preserved by surjective bounded morphisms. Thus if there is a surjective bounded morphism from $\mathcal{F}$ onto $\mathcal{F}^{\prime}$, and $\mathcal{F} \models A$, then $\mathcal{F}^{\prime} \models A$.

\footnotetext{
${ }^{3}$ This means that each member of the modal algebra based on $P$ is the denotation of a constant term. Equivalently, it means that this algebra is generated by its empty subset, so is "zero-generated".
} 


\begin{tabular}{c|c|c|c}
$\mathcal{F}_{\circ}^{\kappa}$ & $\mathcal{F}_{\circ}$ & $\mathcal{F}_{\bullet}^{\kappa}$ & $\mathcal{F}_{\bullet}$ \\
\hline \hline$\circ=\kappa$ & $\circ$ & $\bullet \models \kappa$ & $\bullet$ \\
\hline$\kappa$ & $\neg \kappa$ & $\kappa$ & $\neg \kappa$ \\
$\square A \leftrightarrow A$ & $\square A \leftrightarrow A$ & $\square A$ & $\square A$
\end{tabular}

Table 1: The four singleton frames

We will also use the notion of a point-generated subframe. If $x$ is a point of frame $\mathcal{F}=(W, R,|\kappa|, P)$, the subframe of $\mathcal{F}$ generated by $x$ is the structure $\mathcal{F}^{x}=\left(W^{x}, R^{x},|\kappa|^{x}, P^{x}\right)$, where $W^{x}=\left\{y \in W: x R^{*} y\right\}$ with $R^{*}$ the reflexivetransitive closure of $R ; R^{x}$ is the restriction of $R$ to $W^{x} ;|\kappa|^{x}=|\kappa| \cap W^{x}$; and $P^{x}=\left\{X \cap W^{x}: X \in P\right\}$. Validity is preserved by this construction: if $\mathcal{F}=A$, then $\mathcal{F}^{x} \models A$.

A logic is any set $L$ of formulas that contains all truth-functional tautologies and is closed under modus ponens and under substitution for variables, i.e. if $A \in L$ then $\sigma A \in L$ for every substitution $\sigma$. We may write $\vdash_{L} A$ when $A \in L$, and say that $A$ is an $L$-theorem. A logic $L$ is consistent if $\perp \notin L$, and is normal if contains the formula $\square(p \rightarrow q) \rightarrow(\square p \rightarrow \square q)$ and is closed under the rule of necessitation, i.e. if $A \in L$ then $\square A \in L$. We use standard names for normal logics, but add $\kappa$ as a subscript to indicate that we mean a logic in the language with $\kappa$. For example, a name like "K $4_{\kappa}$ " refers to the set of formulas in the language with $\kappa$ that is generated by the usual axiom schema and rules for K4; whereas "K4" refers to this logic in the language without $\kappa$, which we may also emphasize by calling it "monomodal K4".

For each frame $\mathcal{F}$, the set $L(\mathcal{F})=\{A: \mathcal{F} \models A\}$ of all formulas valid in $\mathcal{F}$ is a consistent normal logic ${ }^{4}$, called the logic determined by $\mathcal{F}$. A logic is quasi-normal if it includes the smallest normal logic K. Quasi-normal logics are characterized by Kripke models and frames with a set of distinguished elements that determine when a formula is true in a model or valid in a frame (see [22, Section III.2]). In particular, for each point $x$ of $\mathcal{F}$, the set $L(\mathcal{F}, x)=\{A$ : $\mathcal{F}, x \models A\}$ of all formulas valid at $x$ in $\mathcal{F}$ is a consistent quasi-normal logic including $L(\mathcal{F})$. Often $L(\mathcal{F}, x)$ is not normal.

A logic $L$ is Post complete if it is maximally consistent, i.e. it is consistent but has no consistent logic properly extending it. A Post complete $L$ has the property that for each closed formula $A$, either $\vdash_{L} A$ or $\vdash_{L} \neg A$. More generally, that property is equivalent to the requirement that a logic $L$ have exactly one Post complete extension [23, Lemma A]. In Table 1 we display some Kripke frames that determine Post complete normal logics. These are the four different one-element Kripke frames that there are, with a name for each in the first row, a graphic representation in the second row, and some formulas they validate in the third (where $A$ is arbitrary). We can take these frames to be based on the set $W=\{0\}$, and we use the common convention that the symbol $\circ$ denotes a

\footnotetext{
${ }^{4}$ Whereas the set $\{A: \mathcal{M} \models A\}$ of all formulas true in a particular model need not be closed under substitution, so may not be a logic.
} 
reflexive point (here $0 R 0$ ), while $\bullet$ denotes an irreflexive one (not $0 R 0$ ). In $\mathcal{F}_{\circ}^{\kappa}$ and $\mathcal{F}_{\bullet}^{\kappa}$ we have $|\kappa|=W$, while in the other two frames $|\kappa|=\emptyset$. Each of these frames is definable: its only subsets are $|\perp|$ and $|T|$. Since each has one element, Post completeness of its logic is then an instance of the following result.

Theorem 2.2. If $\mathcal{F}$ is a definable frame, then for each point $x$ of $\mathcal{F}$, the logic $L(\mathcal{F}, x)$ is Post complete.

Proof. If $L$ is any logic properly extending $L(\mathcal{F}, x)$, then there is a formula $A \in L$ with $\mathcal{F}, x \not \forall A$. Hence $\mathcal{M}, x \not \models A$ for some model $\mathcal{M}$ on $\mathcal{F}$. Since $\mathcal{F}$ is definable, by the proof of Lemma 2.1 there is a closed substitution $\sigma$ with $|A|^{\mathcal{M}}=|\sigma A|$. Hence $\mathcal{F}, x \mid \forall \sigma A$. Then $\mathcal{F}, x \models \neg \sigma A$, so $\neg \sigma A \in L$. But $\sigma A \in L$ by closure under substitution, so $L$ is inconsistent. This proves that $L(\mathcal{F})$ has no consistent proper extension.

Remark 2.3. Post's doctoral dissertation [20] established that the propositional system of Principia Mathematica is semantically complete: every tautology is provable in the system. From this he deduced the result that became known as Post completeness, namely that any formula not provable in the system is inconsistent with it. His argument was that from a non-provable $A$ we can obtain a substitution instance $\sigma A$ whose negation $\neg \sigma A$ is provable. This proof-strategy is essentially that of Theorem 2.2, and indicates the relevance of Lemma 2.1 to Post completeness. Indeed, in Post's case we have that $A$ is not a tautology, so has a falsifying truth-value assignment. Putting $\sigma p=\top$ if $p$ is true under this assignment, and $\sigma p=\perp$ otherwise, defines a closed $\sigma$ for which $\neg \sigma A$ is a tautology, hence provable.

A logic will be called maximally normal if it is consistent and normal but has no consistent normal logic properly extending it. Williamson [28] gave a prooftheoretic characterization of maximal normality for logics that are bimodal (i.e. have two one-place modalities). For the present language, this characterization takes the following slightly simpler form:

Theorem 2.4. A consistent normal logic $L$ is maximally normal iff, for all formulas A,

(1) if $\nvdash_{L} A$, then $\nvdash_{L} \sigma A$ for some closed substitution $\sigma$; and

(2) if $A$ is closed and $\nvdash_{L} A$, then $\vdash_{L} \neg\left(\square^{n_{1}} A \wedge \cdots \wedge \square^{n_{j}} A\right)$ for some natural numbers $j, n_{1}, \ldots, n_{j}$.

Proof. This proceeds essentially as in [28, Proposition 6], but we give the main points. Assuming (1) and (2) hold, let $L^{\prime}$ be any normal proper extension of $L$. Then there is some $A \in L^{\prime}-L$. By (1), $\nvdash_{L} \sigma A$ for some closed $\sigma$. Then by (2) for $\sigma A$, there are numbers $j, n_{1}, \ldots n_{j}$ such that the formula $\neg\left(\square^{n_{1}} \sigma A \wedge\right.$ $\cdots \wedge \square^{n_{j}} \sigma A$ ) is in $L$, hence in $L^{\prime}$. But as $A \in L^{\prime}$ and $L^{\prime}$ is a normal logic, $\vdash_{L^{\prime}} \square^{n_{1}} \sigma A \wedge \cdots \wedge \square^{n_{j}} \sigma A$, so then $L^{\prime}$ is inconsistent. This proves that $L$ is maximally normal. 
Conversely, assume $L$ maximally normal. Let

$$
L^{\prime}=\left\{A: \vdash_{L} \sigma A \text { for every closed substitution } \sigma\right\} .
$$

Then $L^{\prime}$ can be shown to be a normal logic extending $L$, and it is consistent because $L$ is. Hence $L=L^{\prime}$ by maximality. Thus if $\nvdash_{L} A$, then $A \notin L^{\prime}$, implying that (1) holds.

For (2), let $A$ be closed and put

$$
L^{\prime}=\left\{B: \vdash_{L} \square^{n_{1}} A \wedge \cdots \wedge \square^{n_{j}} A \rightarrow B \text { for some } j, n_{1}, \ldots n_{j}\right\} .
$$

Then $L^{\prime}$ is a normal extension of $L$ (the proof that $L^{\prime}$ is closed under substitution depends on $A$ being a closed formula). Moreover $A \in L^{\prime}$. Thus if $\nvdash_{L} A$, then $L^{\prime}$ is a proper normal extension of $L$, hence is inconsistent, so $\perp \in L^{\prime}$. This implies (2).

Note that condition (1) of this Theorem always holds when $L$ is the logic $L(\mathcal{F})$ determined by a definable frame $\mathcal{F}$, as shown in Lemma 2.1 (see Remark $2.3)$.

\section{Kripke-Incomplete Maximal Normality}

French [9] exhibited denumerably many maximally normal logics in the present language, each being defined as the logic determined by some Kripke frame. He asked if there are non-denumerably many maximally normal logics. In this section we give a positive answer by constructing a continuum $\left(2^{\aleph_{0}}\right)$ of maximally normal logics, and moreover show that none of them is determined by any class of Kripke frames. In fact our examples are 'anti-complete' in the sense that none of them is validated by any Kripke frame whatsoever.

Let $\mathbb{N}=\{0,1, \ldots\}$ be the set of natural numbers. The letters $n, m$ will be reserved for members of $\mathbb{N}$. Put $W=\mathbb{N} \cup\{\omega, \kappa\}$, with $\{\omega, \kappa\}$ disjoint from $\mathbb{N}$. If $S$ is any nonempty subset of $\mathbb{N}$, define a relation $R_{S} \subset W \times W$ by putting $R_{S}=I \cup R_{1} \cup R_{2} \cup R_{3} \cup R_{4}$, where:

- $I$ is the identity relation on $W$.

- $R_{1} \subset \mathbb{N}^{2}$ is the immediate predecessor relation $\{(n+1, n): n \in \mathbb{N}\}$.

- $R_{2}=\{(n, \kappa),(\kappa, n): n \in \mathbb{N}\}$.

- $R_{3}=\{(\kappa, \omega),(0, \omega)\}$

- $R_{4}=\{(\omega, n): n \in S\}$.

$\left(W, R_{S}\right)$ is depicted graphically in Figure 1.

Let $P_{W}$ be the Boolean set algebra of all finite or cofinite subsets of $W$. Then $P_{W}$ is closed under the operation $\left[R_{S}\right]$ induced by $R_{S}$. For we have $\left[R_{S}\right] X \subseteq X$ since $R_{S}$ is reflexive, so $\left[R_{S}\right] X$ is finite if $X$ is. Also if $\kappa \notin X$, then $\left[R_{S}\right] X \subseteq\{\omega\}$. 


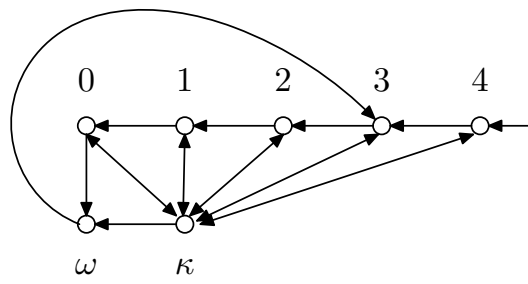

Figure 1: $\left(W, R_{S}\right)$ with $3 \in S$

And if $\kappa \in X$ and $X$ is cofinite, then $\left[R_{S}\right] X$ includes $\{m: n \leq m\}$ for some $n \in \mathbb{N}$. Thus we may define a general frame by putting $\mathcal{F}_{S}=\left(W, R_{S},|\kappa|, P_{W}\right)$, with $|\kappa|=\{\kappa\}$.

Each point $x \in W$ is definable in $\mathcal{F}_{S}$ in the sense that there is a closed formula $\underline{x}$ such that in general, $y=\underline{x}$ iff $y=x$. For these we can take $\underline{\omega}=\square \neg \kappa, \underline{\kappa}=\kappa$, $\underline{0}=\diamond \underline{\omega} \wedge \neg(\underline{\omega} \vee \underline{\kappa})$, and inductively, for each $n \in \mathbb{N}, \underline{n+1}=\diamond \underline{n} \wedge \neg(\underline{\omega} \vee \underline{\kappa} \vee \underline{n})$. It follows that each finite or cofinite subset of $W$ is definable by a closed formula (a Boolean combination of $\underline{x}$ 's), and so $\mathcal{F}_{S}$ is a definable frame.

Lemma 3.1. (1) $x R_{S}^{3} y$ for all $x, y \in W$.

(2) If $A$ is a closed formula and $\mathcal{F}_{S} \not \models A$, then $\mathcal{F}_{S} \models \neg \square^{3} A$.

Proof. (1) Since $R_{S}$ is reflexive, it suffices to show that either $x R_{S} y$ or $x R_{S}^{2} y$ or $x R_{S}^{3} y$.

For the case $x=\omega$, let $m$ be a member of $S$ (which was assumed nonempty). Then $\omega R_{S} \omega, \omega R_{S} m R_{S} \kappa$, and $\omega R_{S} m R_{S} \kappa R_{S} n$ for all $n \in \mathbb{N}$. For the case $x=m \in \mathbb{N}$ we have $m R_{S} \kappa, m R_{S} \kappa R_{S} \omega$, and $m R_{S} \kappa R_{S} n$ for all $n \in \mathbb{N}$. For the case $x=\kappa$, we have $\kappa R_{S} y$ in general.

(2) If $\mathcal{F}_{S} \not \models A$, then $y \not \models A$ for some $y$ in $\mathcal{F}_{S}$. So for any $x$ we get $x \not \models \square^{3} A$ as $x R_{S}^{3} y$, hence $x \models \neg \square^{3} A$. The argument is model-independent as $A$ is closed.

Corollary 3.2. The logic $L\left(\mathcal{F}_{S}\right)$ determined by $\mathcal{F}_{S}$ is maximally normal.

Proof. It suffices to show that $L\left(\mathcal{F}_{S}\right)$ satisfies the two conditions of Theorem 2.4. But condition (1) is given by Lemma 2.1 as $\mathcal{F}_{S}$ is a definable frame, and condition (2) is given by part (2) of the Lemma just proved, with $j=1$ and $n_{1}=3$.

Next we show that the logics $L\left(\mathcal{F}_{S}\right)$ provide $2^{\aleph_{0}}$ examples of maximal normality, by showing that if $S$ and $S^{\prime}$ are distinct non-empty subsets of $\mathbb{N}$, then $L\left(\mathcal{F}_{S}\right) \neq L\left(\mathcal{F}_{S^{\prime}}\right)$. In $\mathcal{F}_{S}$, for all $n \in \mathbb{N}$ we have $\omega R_{S} n$ iff $n \in S$, and so $\mathcal{F}_{S}=\underline{\omega} \rightarrow \diamond \underline{n}$ iff $n \in S$. Thus if $S \neq S^{\prime}$, then there is some $n$ in one of $S$ and $S^{\prime}$ but not in the other, so $\underline{\omega} \rightarrow \diamond \underline{n}$ belongs to one of $L\left(\mathcal{F}_{S}\right)$ and $L\left(\mathcal{F}_{S^{\prime}}\right)$ but not the other, hence $L\left(\mathcal{F}_{S}\right) \neq L\left(\mathcal{F}_{S^{\prime}}\right)$. 
Notice also that in general $\mathcal{F}_{S}, \omega \models \diamond \underline{n}$ iff $n \in S$, and so if $S \neq S^{\prime}$, then $L\left(\mathcal{F}_{S}, \omega\right) \neq L\left(\mathcal{F}_{S^{\prime}}, \omega\right)$. Each logic $L\left(\mathcal{F}_{S}, \omega\right)$ is non-normal, since it contains $\underline{\omega}$ but not $\square \underline{\omega}$, and is Post complete by Theorem 2.2, since $\mathcal{F}_{S}$ is definable. Thus the $L\left(\mathcal{F}_{S}, \omega\right)$ 's are a family of $2^{\aleph_{0}}$ quasi-normal Post complete logics that are not normal. This phenomenon does not occur in monomodal logic, where there are just the two quasi-normal Post complete logics Triv and Ver, and they are both normal.

We turn now to showing that each logic $L\left(\mathcal{F}_{S}\right)$ is Kripke frame incomplete. In fact we show the stronger fact that there no Kripke frames at all that validate $L\left(\mathcal{F}_{S}\right)$. This cannot happen in monomodal logic, where each consistent normal logic is valid in one of the singleton frames.

Define a one-place operation $t^{\mathcal{F}}$ on the powerset of any frame $\mathcal{F}$ by putting

$$
t^{\mathcal{F}} X=\langle R\rangle X-\left(X \cup|\underline{\omega}|^{\mathcal{F}} \cup|\underline{\kappa}|^{\mathcal{F}}\right) .
$$

We will just write $t$ for this term function, allowing the context to indicate what frame $\mathcal{F}$ is intended. In the case that $\mathcal{F}$ is $\mathcal{F}_{S}$ we get that

$$
\begin{aligned}
t X & =\left\langle R_{S}\right\rangle X-(X \cup\{\omega, \kappa\}) \\
& =\left\{n \in(\mathbb{N}-X): \exists y \in X\left(n R_{S} y\right)\right\} .
\end{aligned}
$$

Then $t X \subseteq \mathbb{N}$ for any $X \subseteq W$.

Lemma 3.3. In the frame $\mathcal{F}_{S}$ :

(1) If $X \subseteq \mathbb{N}$, then $n \in t X$ implies $n \geq 1$ and $n-1 \in X$.

(2) If $t(t X) \cup\{0\}=X$, then $X$ is the set $\{2 n: n \in \mathbb{N}\}$ of all even numbers.

Proof. (1) If $n \in t X$, then $n \notin X$ and there is some $y$ with $n R_{S} y \in X \subseteq \mathbb{N}$. Then $n \neq y \in \mathbb{N}$ so this can only mean that $n=y+1$ and $n-1=y \in X$.

(2) Assume $t(t X) \cup\{0\}=X$. Then as $t(t X) \subseteq \mathbb{N}$ we get $X \subseteq \mathbb{N}$. Now every member of $X$ must be even, for otherwise there would be a smallest odd number $n$ in $X$. But then $n \in t(t X)$ so applying (1) twice we get $n-1 \in t X$ and then $n-2 \in X$, contradicting the definition of $n$.

Thus every member of $X$ is even. Hence for any $n$ we have $2 n+1 \notin X$, so $2 n+2 \notin t X$ by (1). Since $(2 n+2) R_{S}(2 n+1) R_{S} 2 n$, it follows that if $2 n \in X$ then $2 n+1 \in t X$ and then $2 n+2 \in t(t X) \subseteq X$. This shows that $2 n \in X$ implies $2 n+2 \in X$. Since $0 \in X$ by assumption, we get that every even number is in $X$ by induction.

Let $T(p)$ be the formula $\nabla p \wedge \neg(p \vee \underline{\omega} \vee \underline{\kappa})$, corresponding to the form of the term function $t$. In any model $\mathcal{M}$ on $\mathcal{F}_{S},|T(p)|^{\mathcal{M}}=t|p|^{\mathcal{M}}$. If $T(T)$ is the result of substituting $T$ for $p$ in $T$, then $|T(T)|^{\mathcal{M}}=t\left(t|p|^{\mathcal{M}}\right)$. 
Theorem 3.4. The formula

$$
\neg \square^{3}(T(T) \vee \underline{0} \leftrightarrow p)
$$

is valid in $\mathcal{F}_{S}$.

Proof. Let $\mathcal{M}$ be any model on $\mathcal{F}_{S}$. Put $X=|p|^{\mathcal{M}} \in P_{W}$. Then $X$ is either finite or cofinite, so by Lemma 3.3(2), $t(t X) \cup\{0\} \neq X$. Thus $|T(T) \vee \underline{0}|^{\mathcal{M}} \neq|p|^{\mathcal{M}}$, so there is a point $y$ at which $T(T) \vee \underline{0} \leftrightarrow p$ is false in $\mathcal{M}$. But for any $x \in W$, we have $x R_{S}^{3} y$ by Lemma 3.1(1), so $\neg \square^{3}(T(T) \vee \underline{0} \leftrightarrow p)$ is true at $x$ in $\mathcal{M}$.

Now let $\mathcal{F}=\left(V, R,|\kappa|^{\mathcal{F}}\right)$ be any Kripke frame. We are going to show that the formula (3.2) is not valid in $\mathcal{F}$, provided that $\mathcal{F}$ preserves the distinction between the 'numerals' $\underline{n}$ by validating $\neg(\underline{n} \wedge \underline{m})$ whenever $n \neq m$. The proof uses the following two subsets of $V$ :

$$
\begin{aligned}
& \mathbb{E}=\bigcup\left\{|\underline{2 n}|^{\mathcal{F}}: n \in \mathbb{N}\right\}, \\
& \mathbb{O}=\bigcup\left\{|\underline{2 n+1}|^{\mathcal{F}}: n \in \mathbb{N}\right\} .
\end{aligned}
$$

We also need the operation $t$ on the powerset of $\mathcal{F}$ defined as in (3.1).

Lemma 3.5. Suppose that $\mathcal{F} \models \neg(\underline{n} \wedge \underline{m})$ for all distinct $n, m \in \mathbb{N}$. Then

(1) $t \mathbb{E}=\mathbb{O}$.

(2) $t \mathbb{O} \cup|\underline{0}|^{\mathcal{F}}=\mathbb{E}$.

(3) $\mathcal{F} \not \models \neg \square^{3}(T(T) \vee \underline{0} \leftrightarrow p)$.

Proof. For (1), if $x \in t \mathbb{E}$, then $x \in\langle R\rangle \mathbb{E}$, so there is a $y$ with $x R y \in \mathbb{E}$, hence $y=\underline{2 n}$ for some $n$. Also $x \notin \mathbb{E} \cup|\underline{\omega}|^{\mathcal{F}} \cup|\underline{\kappa}|^{\mathcal{F}}$, so $x \notin|\underline{2 n}|^{\mathcal{F}}$ and altogether the formula $\vee \underline{2 n} \wedge \neg(\underline{\omega} \vee \underline{\kappa} \vee \underline{2 n})$ is true at $x$. But this formula is $\underline{2 n+1}$ by definition, so $x \in|\underline{2 n+1}|^{\mathcal{F}} \subseteq \mathbb{O}$.

This proves that $t \mathbb{E} \subseteq \mathbb{O}$. For the converse inclusion, if $x \in|2 n+1|^{\mathcal{F}}$ for some $n$, then $x \models \diamond \underline{2 n} \wedge \neg(\underline{\omega} \vee \underline{\kappa} \vee \underline{2 n})$, so $x \in\langle R\rangle \mathbb{E}-\left(|\underline{\omega}|^{\mathcal{F}} \cup|\underline{\kappa}|^{\mathcal{F}}\right)$. It remains to show that $x \notin \mathbb{E}$ to conclude that $x \in t \mathbb{E}$. But $x \models 2 n+1$, while $\mathcal{F}$ validates $\neg(\underline{2 n+1} \wedge \underline{2 m})$ in general by assumption, so $x \notin|\underline{2 m}|^{\mathcal{F}}$ for any $m \in \mathbb{N}$ as required.

That completes the proof of (1). The proof of (2) is similar.

For (3), let $\mathcal{M}$ be a model on $\mathcal{F}$ with $|p|^{\mathcal{M}}=\mathbb{E}$. From (1) and (2) we have $t(t \mathbb{E}) \cup|\underline{0}|^{\mathcal{F}}=\mathbb{E}$, giving $|T(T) \vee \underline{0}|^{\mathcal{M}}=|p|^{\mathcal{M}}$. It follows that $\mathcal{M}$ verifies $\square^{3}(T(T) \vee \underline{0} \leftrightarrow p)$ rather than its negation, so $\mathcal{F}$ invalidates this negation.

Theorem 3.6. If $S$ is any non-empty subset of $\mathbb{N}$, then the logic $L\left(\mathcal{F}_{S}\right)$ is not valid in any Kripke frame.

Proof. Let $\mathcal{F}$ be any Kripke frame. Suppose, for the sake of contradiction, that $\mathcal{F}$ validated $L\left(\mathcal{F}_{S}\right)$. Then since $\neg(\underline{n} \wedge \underline{m})$ is valid in $\mathcal{F}_{S}$ it would be valid in $\mathcal{F}$, for all distinct $n, m$. Hence by Lemma 3.5(3) the formula (3.2) would not be valid in $\mathcal{F}$. But this formula belongs to $L\left(\mathcal{F}_{S}\right)$ by Theorem 3.4 , so would be valid in $\mathcal{F}$, a contradiction. 
The Kripke frame incompleteness of $L\left(\mathcal{F}_{S}\right)$ can now be formulated as the observation that there are many non-theorems of $L\left(\mathcal{F}_{S}\right)$ (including $\perp$ ), but none of them can be falsified by a model on a Kripke frame for $L\left(\mathcal{F}_{S}\right)$, since there are no such frames.

In passing we note that this 'anti-completeness' can be strengthened in the context of algebraic semantics for the present language. This uses a type of algebra $\mathbf{A}$ comprising a Boolean algebra with an operation interpreting $\square$ and a distinguished element interpreting $\kappa$. Each formula $B$ with $n$ variables induces an $n$-ary operation $B^{\mathbf{A}}$ on $\mathbf{A}$, with $B^{\mathbf{A}}$ being a distinguished element (nullary operation) in the case that $B$ is closed. A validates $B$ when it satisfies the equation $B^{\mathbf{A}}=1$. A logic $L$ gives rise to the variety (equational class) $\mathcal{V}(L)$ of algebras validating all $L$-theorems.

An algebra $\mathbf{A}$ is complete if each of its subsets has a join (least upper bound). Litak [17] exhibited a tense logic whose associated variety contains no non-trivial complete algebra. The powerset algebra of a Kripke frame $\mathcal{F}$ is a complete algebra of this kind in which $\square$ is interpreted as the operation $[R]$ and $\kappa$ is interpreted as $|\kappa|^{\mathcal{F}}$. This algebra is also atomic, and is completely additive, i.e. the operation interpreting $\diamond$ preserves all joins. Conversely, every complete atomic and completely additive algebra is isomorphic to the powerset algebra of a Kripke frame. ${ }^{5}$ Moreover, it can be shown from work of Kowalski and Kracht $\left[15\right.$, Proposition 6] that every algebra in the variety $\mathcal{V}\left(L\left(\mathcal{F}_{S}\right)\right)$ is completely additive. Therefore, Theorem 3.6 is equivalent to the statement that the variety $\mathcal{V}\left(L\left(\mathcal{F}_{S}\right)\right)$ contains no non-trivial complete and atomic algebras. The strengthening is that, by adapting the above arguments, we can show that $\mathcal{V}\left(L\left(\mathcal{F}_{S}\right)\right)$ contains no non-trivial complete algebras, not even non-atomic ones. A complete algebra $\mathbf{A}$ has the elements $\bigvee\left\{|\underline{2 n}|^{\mathbf{A}}: n \in \mathbb{N}\right\}$ and $\bigvee\left\{|\underline{2 n+1}|^{\mathbf{A}}: n \in \mathbb{N}\right\}$, where $\bigvee$ denotes the join operation in $\mathbf{A}$, and these elements play a role parallel to $\mathbb{E}$ and $\mathbb{O}$ in showing that if $\mathbf{A}$ were a non-trivial complete member of $\mathcal{V}\left(L\left(\mathcal{F}_{S}\right)\right)$ it would invalidate formula $(3.2)$.

\section{Weak Transitivity}

Which logics have continuum many maximally normal extensions? One immediate answer is that each frame $\mathcal{F}_{S}$ has reflexive $R_{S}$ and so validates the axiom $\mathrm{T}: \square p \rightarrow p$. Thus all of the maximally normal logics $L\left(\mathcal{F}_{S}\right)$ are extensions of $\mathrm{KT}_{\kappa}$.

Another answer concerns the axiom $4^{2}: \diamond^{3} p \rightarrow \diamond^{2} p$, which corresponds to the 2-transitivity frame condition that $x R^{3} y$ implies $x R^{2} y$.

Lemma 4.1. If $S$ contains all even numbers, then $\mathcal{F}_{S}$ is 2-transitive and so validates $4^{2}$.

Proof. Let $S$ contain all even numbers. Then we show that $x R_{S}^{2} y$ for all $x, y$ in $\mathcal{F}_{S}$, implying 2-transitivity. From the proof of Lemma 3.1(1), the only case

\footnotetext{
${ }^{5} \mathrm{~A}$ fact that ultimately goes back to [13].
} 


\begin{tabular}{c|c|c|c}
$\mathcal{F}_{\circ \circ}$ & $\mathcal{F}_{\bullet \bullet}$ & $\mathcal{F}_{\bullet \circ}$ & $\mathcal{F}_{\circ}$ \\
\hline \hline$\circ \leftrightarrow \circ$ & $\bullet \leftrightarrow \bullet$ & $\bullet \leftrightarrow \circ$ & $\circ \leftrightarrow \bullet$ \\
\hline$\diamond \kappa$ & $\nabla \kappa \leftrightarrow \neg \kappa$ & $\diamond \kappa$ & $\diamond \kappa \leftrightarrow \neg \kappa$ \\
$\diamond \neg \kappa$ & $\diamond \neg \kappa \leftrightarrow \kappa$ & $\diamond \neg \kappa \leftrightarrow \kappa$ & $\diamond \neg \kappa$
\end{tabular}

Table 2: Four weakly transitive doubleton frames

where $x R_{S}^{2} y$ might fail is when $x=\omega$ and $y=n$ for some $n$. But now we have $\omega R_{S} n$ if $n$ is even, and $\omega R_{S}(n+1) R_{S} n$ otherwise.

Since there are continuum many subsets of $\mathbb{N}$ that contain all even numbers, it follows that there are continuum many maximally normal logics of the form $L\left(\mathcal{F}_{S}\right)$ that extend $\mathrm{K} 4_{\kappa}^{2}$.

For logics containing the transitivity axiom $4: \diamond^{2} p \rightarrow \diamond p$, the situation differs rather dramatically. There are exactly five maximally normal extensions of $\mathrm{K} 4_{\kappa}$, namely the logics determined by the four Kripke frames of Table 1 and the frame $\mathcal{F}_{\circ \circ}$ of the first column of Table 2. In this new table, all four frames have two elements, and we can take them all to be based on $W=\{0,1\}$, with 0 on the left and 1 on the right in the graphic representations in the second row. All have $0 R 1$ and $1 R 0$, with the rest of $R$ determined by whether or not 0 and 1 are reflexive, as indicated by the symbols $\circ$ and $\bullet$ in the diagrams and in the names of the frames. In each frame we define $|\kappa|=\{1\}$, i.e. $\kappa$ is true just at the right-hand point. The logics of these frames are all maximally normal by Theorem 2.4. Condition (1) of that Theorem holds for each logic as these frames are all definable, and condition (2) holds because, in each case, if a closed formula $A$ is not valid on the frame, then $\neg(A \wedge \square A)$ is valid. But unlike the singleton frames, the logics of these doubletons are not Post complete, since they include neither $\kappa$ nor $\neg \kappa$ as a theorem.

$\mathrm{S} 4_{\kappa}\left(=\mathrm{KT} 4_{\kappa}\right)$ has exactly three maximally normal extensions: the logics of the frames $\mathcal{F}_{\circ}^{\kappa}, \mathcal{F}_{\circ}$ and $\mathcal{F}_{\circ \circ}$. In Table $2, \mathcal{F}_{\circ \circ}$ is transitive, while $\mathcal{F}_{\bullet \bullet}, \mathcal{F}_{\bullet \circ}$ and $\mathcal{F}_{\circ} \bullet$ are not. But all four are weakly transitive, which means that if $x R^{2} y$ and $x \neq y$, then $x R y$. This frame condition corresponds to the axiom $4^{w}: \diamond^{2} p \rightarrow p \vee \diamond p$. The monomodal logic $\mathrm{K} 4^{w}$ is the modal logic resulting from the interpretation of $\diamond$ as the topological derivative (set of limit points). This has been extensively studied under the name wK4 by researchers associated with the Georgian school $[6,3]$. There are exactly eight maximally normal extensions of $\mathrm{K} 4_{\kappa}^{w}$, namely the logics determined by the eight frames of Tables 1 and 2 .

To prove these claims requires a proof-theoretic analysis. First we give a result that will provide characterizations of the five maximally normal transitive logics.

Theorem 4.2. Let $L$ be a consistent normal logic.

(1) If $\nvdash_{L} \diamond \top$, then $L$ is a sublogic of either $L\left(\mathcal{F}_{\bullet}^{\kappa}\right)$ or $L\left(\mathcal{F}_{\bullet}\right)$.

(2) If $\vdash_{L} \diamond \top$, then: 
(i) If $\vdash_{L} \kappa$, then $L$ is a sublogic of $L\left(\mathcal{F}_{\circ}^{\kappa}\right)$;

(ii) If $\vdash_{L} \neg \kappa$, then $L$ is a sublogic of $L\left(\mathcal{F}_{\circ}\right)$;

(iii) If $\nvdash_{L} \kappa$ and $\nvdash_{L} \neg \kappa$, while $\vdash_{L} \diamond \kappa$ and $\vdash_{L} \diamond \neg \kappa$, then $L$ is a sublogic of $L\left(\mathcal{F}_{\circ \circ}\right)$.

Proof. Let $\mathcal{F}_{L}=\left(W_{L}, R_{L},|\kappa|_{L}, P_{L}\right)$ be the canonical general frame of $L$, based on the set $W_{L}$ of maximally $L$-consistent sets of formulas, with the relation $R_{L}$ having $x R_{L} y$ iff $\{A: \square A \in x\} \subseteq y$, and $P_{L}$ being the set of all subsets of the form $|A|_{L}=\left\{x \in W_{L}: A \in x\right\}$ for all formulas $A$. We assume the standard theory of $\mathcal{F}_{L}$, including the fact that it validates $L$, and that $\vdash_{L} A$ iff $|A|_{L}=W_{L}$.

For (1), if $\nvdash_{L} \diamond \top$, then there is an $x \in W_{L}$ with $\diamond \top \notin x$. Therefore $\left\{y: x R_{L} y\right\}=\emptyset$, so the subframe $\mathcal{F}_{L}^{x}$ of $\mathcal{F}_{L}$ generated by $x$ is an irreflexive frame based on the singleton $\{x\}$. Both subsets are admissible, so $\mathcal{F}_{L}^{x}$ can be viewed as a Kripke frame. If $x \in|\kappa|_{L}$, then $|\kappa|_{L}^{x}=\{x\}$ and $\mathcal{F}_{L}^{x}$ is an isomorphic copy of $\mathcal{F}_{\bullet}^{\kappa}$. Since validity of formulas is preserved in passing from $\mathcal{F}_{L}$ to $\mathcal{F}_{L}^{x}$, this implies that $L \subseteq L\left(\mathcal{F}_{\bullet}^{\kappa}\right)$. If however $x \notin|\kappa|_{L}$, then $\mathcal{F}_{L}^{x}$ is a copy of $\mathcal{F}_{\bullet}$, and so $L \subseteq L\left(\mathcal{F}_{\bullet}\right)$.

For case (2)(i), let $\vdash_{L} \diamond \top$ and $\vdash_{L} \kappa$. Then $R_{L}$ is a serial relation on $W_{L}$, i.e. $\forall x \exists y\left(x R_{L} y\right)$, and $|\kappa|_{L}=W_{L}$. This ensures that the unique map $f_{0}: W_{L} \rightarrow\{0\}$ is a bounded morphism from $\mathcal{F}_{L}$ onto $\mathcal{F}_{\circ}^{\kappa}$. Since surjective bounded morphisms preserve validity, $f_{0}$ thus ensures that $L$ is valid in $\mathcal{F}_{\circ}^{\kappa}$, showing that $L \subseteq L\left(\mathcal{F}_{\circ}^{\kappa}\right)$.

For case (2)(ii), if $\vdash_{L} \diamond \top$ and $\vdash_{L} \neg \kappa$, then $|\kappa|_{L}=\emptyset=f_{0}^{-1}|\kappa|^{\mathcal{F}_{\circ}}$, where $f_{0}$ is as in case $2(\mathrm{i})$, and $f_{0}$ becomes a bounded morphism from $\mathcal{F}_{L}$ onto $\mathcal{F}_{\mathrm{o}}$, leading to $L \subseteq L\left(\mathcal{F}_{\circ}\right)$.

For case (2)(iii), it suffices to show that there is a surjective bounded morphism from $\mathcal{F}_{L}$ onto $\mathcal{F}_{\circ \circ}$. For this we define $f_{\kappa}: W_{L} \rightarrow\{0,1\}$ to be the characteristic function of $|\kappa|_{L}$. Thus $f_{\kappa}^{-1}|\kappa|^{\mathcal{F}_{\circ \circ}}=f_{\kappa}^{-1}\{1\}=|\kappa|_{L}$; while $f_{\kappa}^{-1}\{0\}$ is equal to the complement $-|\kappa|_{L}$. This implies that $f_{\kappa}$ is $\kappa$-invariant, and also that it reflects admissibility.

Now if $\nvdash_{L} \kappa$ and $\nvdash_{L} \neg \kappa$, then both $-|\kappa|_{L}$ and $|\kappa|_{L}$ are non-empty, so $f_{\kappa}$ is surjective. If further $\vdash_{L} \diamond \kappa$ and $\vdash_{L} \diamond \neg \kappa$, then in $\mathcal{F}_{L},\left\langle R_{L}\right\rangle|\kappa|_{L}=\left\langle R_{L}\right\rangle\left(-|\kappa|_{L}\right)=$ $W_{L}$, so every $x \in W_{L}$ is $R_{L}$-related to a point in $|\kappa|_{L}$, and to a point in $-|\kappa|_{L}$. Since $R$ is universal in $\mathcal{F}_{\circ \circ}$, this is enough to ensure that $f_{\kappa}$ satisfies the back and forth conditions, and so is a bounded morphism onto $\mathcal{F}_{\circ \circ}$ as required.

\section{Corollary 4.3.}

(1) $L\left(\mathcal{F}_{\bullet}^{\kappa}\right)$ is the unique maximally normal logic containing $\kappa$ but not $\diamond \top$.

(2) $L\left(\mathcal{F}_{\bullet}\right)$ is the unique maximally normal logic containing $\neg \kappa$ but not $\diamond \top$.

(3) $L\left(\mathcal{F}_{\circ}^{\kappa}\right)$ is the unique maximally normal logic containing $\diamond \top$ and $\kappa$.

(4) $L\left(\mathcal{F}_{\circ}\right)$ is the unique maximally normal logic containing $\diamond \top$ and $\neg \kappa$.

(5) $L\left(\mathcal{F}_{\circ \circ}\right)$ is the unique maximally normal logic containing $\diamond \kappa$ and $\diamond \neg \kappa$ but not $\kappa$ or $\neg \kappa$. 
Lemma 4.4. Let $L$ be a maximally normal extension of $K{ }_{4}{ }_{\kappa}{ }_{\kappa}$ and $A$ any closed formula.

(1) $\nvdash_{L} A$ implies $\vdash_{L} A \rightarrow \diamond \neg A$.

(2) $\nvdash_{L} \neg A$ implies $\vdash_{L} \neg A \rightarrow \diamond A$.

(3) If $\nvdash_{L} A$ and $\nvdash_{L} \neg A$, then

(i) $\nvdash \diamond A$ implies $\vdash_{L} \diamond A \leftrightarrow \neg A$, and

(ii) $\nvdash \diamond \neg A$ implies $\vdash_{L} \diamond \neg A \leftrightarrow A$.

Proof. Using axiom $4^{w}$ we can obtain $\vdash_{L} \diamond(B \vee \diamond B) \rightarrow B \vee \diamond B$, for any $B$. This is then used to show by induction that

$$
\vdash_{L} \diamond^{n} B \rightarrow B \vee \diamond B \quad \text { for all } n \geq 0 .
$$

For (1), if $\nvdash_{L} A$ then by Theorem 2.4(2) there are $j, n_{1}, \ldots, n_{j}$ such that

$$
\vdash_{L} \diamond^{n_{1}} \neg A \vee \cdots \vee \diamond^{n_{j}} \neg A .
$$

But $\vdash_{L} \diamond^{n_{i}} \neg A \rightarrow \neg A \vee \diamond \neg A$ for all $i$ by (4.1), so this leads by Boolean logic to $\vdash_{L} \neg A \vee \diamond \neg A$, hence $\vdash_{L} A \rightarrow \diamond \neg A$.

(2) follows from (1) by replacing $A$ by $\neg A$.

For (3)(i), suppose first that $\nvdash_{L} \diamond A \rightarrow \neg A$. Then $\nvdash_{L} \neg(\diamond A \wedge A)$ so replacing $A$ by $\diamond A \wedge A$ in (2) gives $\vdash_{L} \neg(\diamond A \wedge A) \rightarrow \diamond(\diamond A \wedge A)$. But $\neg \diamond A \rightarrow \neg(\diamond A \wedge A)$ is a tautology, and $\diamond(\diamond A \wedge A) \rightarrow \diamond A$ belongs to any normal logic, so from all these we obtain $\vdash_{L} \neg \diamond A \rightarrow \diamond A$, hence $\vdash_{L} \diamond A$.

Contrapositively then, if $\nvdash_{L} \diamond A$, then $\vdash_{L} \diamond A \rightarrow \neg A$. But if also $\nvdash_{L} \neg A$, then $\vdash_{L} \neg A \rightarrow \diamond A$ by (2), and altogether $\vdash_{L} \diamond A \leftrightarrow \neg A$. So 3(i) holds.

(3)(ii) follows from 3 (i), replacing $A$ by $\neg A$.

Theorem 4.5. If $L$ is a maximally normal extension of $K 4_{\kappa}^{w}$, then $L$ is the logic determined by one of the frames $\mathcal{F}_{\circ}^{\kappa}, \mathcal{F}_{\circ}, \mathcal{F}_{\bullet}^{\kappa}, \mathcal{F}_{\bullet}, \mathcal{F}_{\circ \circ}, \mathcal{F}_{\bullet \bullet}, \mathcal{F}_{\bullet \circ}, \mathcal{F}_{\circ} \bullet$

Proof. Suppose that $L$ is not determined by any of the first five frames on the list. Then by Theorem 4.2 and the maximality of $L$ we must have $\vdash_{L} \diamond \top, \nvdash_{L} \kappa$ and $\nvdash_{L} \neg \kappa$, but not both $\vdash_{L} \diamond \kappa$ and $\vdash_{L} \diamond \neg \kappa$. This leaves three cases:

Case 1: $\nvdash_{L} \diamond \kappa$ and $\nvdash_{L} \diamond \neg \kappa$. Then putting $A=\kappa$ in Lemma 4.4(3) gives $\vdash_{L} \diamond \kappa \leftrightarrow \neg \kappa$ and $\vdash_{L} \diamond \neg \kappa \leftrightarrow \kappa$. Thus $\left\langle R_{L}\right\rangle|\kappa|_{L}=-|\kappa|_{L}$ and $\left\langle R_{L}\right\rangle-|\kappa|_{L}=|\kappa|_{L}$, so the points in $W_{L}$ that are $R_{L}$-related to a point of $|\kappa|_{L}$ are precisely those in the complement $-|\kappa|_{L}$, and vice versa with $|\kappa|_{L}$ and $-|\kappa|_{L}$ interchanged. This implies that the map $f_{\kappa}$ of the proof of case (2)(iii) of Theorem 4.2 has the back

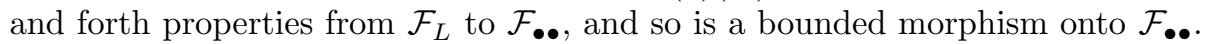
This is enough to make $L \subseteq L\left(\mathcal{F}_{\bullet \bullet}\right)$, hence $L=L\left(\mathcal{F}_{\bullet \bullet}\right)$ by maximality.

Case 2: $\vdash_{L} \diamond \kappa$ and $\nvdash_{L} \diamond \neg \kappa$. Then $\vdash_{L} \diamond \neg \kappa \leftrightarrow \kappa$ by Lemma 4.4(3)(ii). Then every $x \in W_{L}$ is $R_{L}$-related to a point in $|\kappa|_{L}$, while the points $R_{L}$-related to a point of $-|\kappa|_{L}$ are precisely those in $|\kappa|_{L}$. This makes $f_{\kappa}$ a bounded morphism onto $\mathcal{F}_{\bullet}$, leading to $L=L\left(\mathcal{F}_{\bullet}\right)$.

Case 3: $\nvdash_{L} \diamond \kappa$ and $\vdash_{L} \diamond \neg \kappa$. Then $\vdash_{L} \diamond \kappa \leftrightarrow \neg \kappa$ and $f_{\kappa}$ is a bounded morphism onto $\mathcal{F}_{\circ} \bullet$, yielding $L=L\left(\mathcal{F}_{\circ} \bullet\right.$. 
Corollary 4.6. If $L$ is a maximally normal extension of $K 4 \kappa$, then $L$ is the logic determined by one of the frames $\mathcal{F}_{\circ}^{\kappa}, \mathcal{F}_{\circ}, \mathcal{F}_{\bullet}^{\kappa}, \mathcal{F}_{\bullet}, \mathcal{F}_{\circ \circ}$.

If $L$ is a maximally normal extension of $S_{4 \kappa}$, then $L$ is the logic determined by one of the frames $\mathcal{F}_{\circ}^{\kappa}, \mathcal{F}_{\circ}, \mathcal{F}_{\circ \circ}$.

\section{Extensions of S4.3}

In the realm of monomodal logics, Bull [4] proved the famous result that every normal extension of S4.3 has the finite model property. Fine [7] gave a complete description of these extensions, showing that there are only $\aleph_{0}$ of them, and that there is no infinite strictly increasing sequence of them, so they are all finitely axiomatizable and decidable. Segerberg [24] later proved that every logic extending S4.3 is normal.

In this section we show that in the presence of a constant $\kappa$, none of these properties hold. First we use $\kappa$ to construct a continuum of normal extensions of S4. $3_{\kappa}$ that lack the finite model property. Related constructions for monomodal extensions of K4 and K4.3 can found in [5, Section 6.1].

Consider the linearly ordered Kripke frame $(\mathbb{N}, \geqslant,\{2 n: n \in \mathbb{N}\})$ in which each point is related to all smaller points and $\kappa$ is true at all even numbers and false at all odd ones. Each point $n$ of this frame is definable by a constant formula $\underline{n}$. The first few in this sequence of formulas are

$$
\begin{aligned}
& \underline{0}=\square \kappa \\
& \underline{1}=\square(\kappa \rightarrow \underline{0}) \wedge \neg \underline{0} \\
& \underline{2}=\square(\neg \kappa \rightarrow \underline{1}) \wedge \neg \underline{0} \wedge \neg \underline{1} \\
& \underline{3}=\square(\kappa \rightarrow \underline{0} \vee \underline{2}) \wedge \neg \underline{0} \wedge \neg \underline{1} \wedge \neg \underline{2} \\
& \underline{4}=\square(\neg \kappa \rightarrow \underline{1} \vee \underline{3}) \wedge \neg \underline{0} \wedge \neg \underline{1} \wedge \neg \underline{2} \wedge \neg \underline{3} \\
& \vdots
\end{aligned}
$$

To define $\underline{n}$ inductively, write $\geqslant n$ for the conjunction $\bigwedge\{\neg \underline{m}: n>m\}$. Put $\underline{0}=\square \kappa$. For $n>0$, if $n$ is odd let $\underline{n}$ be

$$
\square(\kappa \rightarrow \underline{0} \vee \underline{2} \vee \cdots \vee \underline{n-1}) \wedge(\underline{\geqslant n}),
$$

and if $n$ is even let $\underline{n}$ be

$$
\square(\neg \kappa \rightarrow \underline{1} \vee \underline{3} \vee \cdots \vee \underline{n-1}) \wedge(\underline{\geqslant n}) .
$$

In this frame one can show by induction on $n$ that, for all $m \in \mathbb{N}$,

$$
m \models \underline{n} \quad \text { iff } \quad m=n .
$$

Now let $X=\{\alpha, \beta\}$, with $\alpha, \beta$ distinct entities not in $\mathbb{N}$, and extend the above frame to the frame

$$
\mathcal{G}=(X \cup \mathbb{N}, R,\{2 n: n \in \mathbb{N}\}),
$$


where $R=(X \times(X \cup \mathbb{N})) \cup\left\{(m, n) \in \mathbb{N}^{2}: m \geqslant n\right\}$. Thus $\alpha$ and $\beta$ are $R$-related to everything, including each other, and so $X$ forms a two-element cluster placed 'to the left' of the ordering $\geqslant$, i.e. $R$ looks like

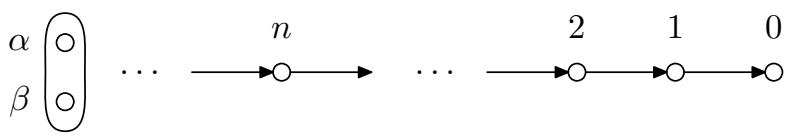

No $m \in \mathbb{N}$ is $R$-related to $\alpha$ or $\beta$, so statement (5.1) continues to hold in $\mathcal{G}$. Moreover, as $\alpha$ and $\beta$ are related to all members of $\mathbb{N}$, no formula $\underline{n}$ is true at $\alpha$ or $\beta$. Hence $\underline{n}$ defines $n$ uniquely in $\mathcal{G}$.

We make use of $G r z$, the well-known axiom $\square(\square(p \rightarrow \square p) \rightarrow p) \rightarrow p$. This cannot be falsified at any $n \in \mathbb{N}$ in $\mathcal{G}$. It can be false at most at $\alpha$ and $\beta$, so for each $n \in \mathbb{N}$ the formula $\neg G r z \rightarrow \diamond \underline{n}$ is valid in $\mathcal{G}$. ( $G r z$ is falsifiable at $\alpha$ by taking $p$ false at $\alpha$ and true everywhere else; and is likewise falsifiable at $\beta$ by taking $p$ false only at $\beta$.)

Let $L_{0}$ be the smallest logic that extends $\mathrm{S} 4.3_{\kappa}$ and contains all the formulas $\{\neg G r z \rightarrow \diamond \underline{n}: n \in \mathbb{N}\}$. As before, let $L(\mathcal{G})$ be the (normal) logic determined by the frame $\mathcal{G}$. Then $L_{0} \subseteq L(\mathcal{G})$, and $\operatorname{Grz} \notin L(\mathcal{G})$.

Theorem 5.1. If $L$ is any logic extending $L_{0}$ and having Grz $\notin L$, then $L$ does not have the finite model property.

Proof. Let $\mathcal{M}$ be any model of $L$. If $G r z$ is not true in $\mathcal{M}$, then $\neg G r z$ is true at some (distinguished) point $x$, and so as $L_{0} \subseteq L$, for each $n \in \mathbb{N}$ we get $\nabla \underline{n}$ true at $x$, hence there is a point $x_{n}$ in $\mathcal{M}$ such that $x_{n} \models \underline{n}$. Then $x_{n} \neq x_{m}$ whenever $n \neq m$; for either $n>m$ and so $\neg \underline{m}$ is a conjunct of $\geqslant n$, hence a conjunct of $\underline{n}$, implying $x_{n} \models \neg \underline{m}$ while $x_{m} \models \underline{m}$; or else $m>n$ and likewise $x_{m} \models \neg \underline{n}$ while $x_{n} \models \underline{n}$. Hence $\overline{\mathcal{M}}$ has infinitely many points.

It follows that every finite model of $L$ must verify $G r z$. But $\operatorname{Grz} \notin L$.

Next, we show that there are continuum many normal logics between $L_{0}$ and $L(\mathcal{G})$. For each $n \in \mathbb{N}$, let $A_{n}$ be the formula

$$
\square(\underline{n} \rightarrow p) \vee \square(\underline{n} \rightarrow \neg p) .
$$

Since $\underline{n}$ is true at exactly one point of $\mathcal{G}, A_{n}$ belongs to $L(\mathcal{G})$.

Let $\mathcal{G}^{n}$ be the modification of $\mathcal{G}$ obtained by inserting a new point $n^{\prime}$ between $n+1$ and $n$ that will also satisfy $\underline{n}$. For this we define $\kappa$ to be true at $n^{\prime}$ iff $n$ is even (so $n$ and $n^{\prime}$ are indistinguishable by $\kappa$ ). The relation $R$ is extended to $\mathcal{G}^{n}$ by requiring $x R n^{\prime}$ iff $x R n$, and $n^{\prime} R x$ iff $n R x$, for each $x \neq n$ in $\mathcal{G}$, and adding $n^{\prime} R n^{\prime} R n$. Thus $\mathcal{G}^{n}$ looks like

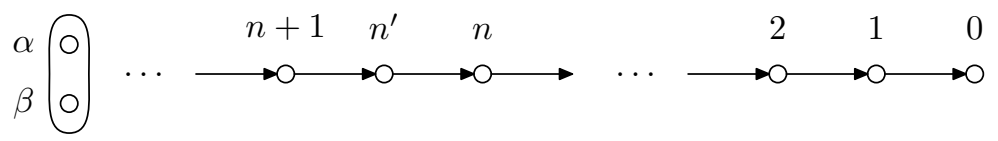

The point of this is that the formula $\underline{n}$ is now true at both $n$ and $n^{\prime}$, so $A_{n}$ is falsified in $\mathcal{G}^{n}$ (at every $x$ such that $x R n^{\prime}$ ) whenever $p$ is true at one of $n$ and 
$n^{\prime}$ and false at the other. On the other hand, if $m \neq n$, then $\underline{m}$ is still true in $\mathcal{G}^{n}$ exactly at $m$, and hence $\mathcal{G}^{n} \models A_{m}$.

Now for each $S \subseteq \mathbb{N}$, define $L_{S}$ to be the smallest normal extension of $L_{0}$ containing $\left\{A_{n}: n \in S\right\}$. Then $L_{0} \subseteq L_{S} \subseteq L(\mathcal{G})$, and so $\operatorname{Grz} \notin L_{S}$. Moreover, if $n \notin S$, then as the frame $\mathcal{G}^{n}$ validates $A_{m}$ for every $m \neq n$, it validates $A_{m}$ for every $m \in S$, but falsifies $A_{n}$, showing that $A_{n} \notin L_{S}$. So for any $n \in \mathbb{N}$,

$$
A_{n} \in L_{S} \quad \text { iff } \quad n \in S
$$

Therefore if $S, S^{\prime} \subseteq \mathbb{N}$ with $S \neq S^{\prime}$, it follows that $L_{S} \neq L_{S^{\prime}}$. The $L_{S}$ 's are thus a family of $2^{\aleph_{0}}$ normal extensions of $S 4.3_{\kappa}$ that lack the finite model property by Theorem 5.1. Since there are only $\aleph_{0}$ decidable logics, this also implies that there are undecidable normal extensions of S4. $3_{\kappa}$. Indeed, (5.2) implies that $L_{S}$ is undecidable whenever $S$ is.

Now taking any infinite strictly increasing sequence $S_{1} \subset S_{2} \subset \ldots$ of subsets of $\mathbb{N}$ yields an infinite strictly increasing sequence $L_{S_{1}} \subset L_{S_{2}} \subset \ldots$ of normal logics between $L_{0}$ and $L(\mathcal{G})$. The union $\bigcup_{1}^{\infty} L_{S_{n}}$ is then a normal logic between $L_{0}$ and $L(\mathcal{G})$ that is not finitely axiomatizable. More concretely, $L_{0}$ is itself a normal extension of S4.3 $3_{\kappa}$ that is not finitely axiomatizable. To see this, let $L_{n}^{\prime}$ be the smallest normal extension of S4.3 $3_{\kappa}$ to include $\{\neg G r z \rightarrow \diamond \underline{m}: m \leq n\}$. Define a finite frame by deleting the points of $\{m \in \mathbb{N}: m>n\}$ from $\mathcal{G}$, leaving only $\alpha, \beta, n, n-1, \ldots, 0$. This frame validates $L_{n}^{\prime}$ but falsifies $\neg G r z \rightarrow \diamond \underline{n+1}$, showing that $L_{n}^{\prime} \subset L_{n+1}^{\prime}$. The $L_{n}^{\prime}$ 's form a strictly increasing sequence whose union is $L_{0}$. If $L_{0}$ were finitely axiomatizable it would be equal to some $L_{n}$, contradicting that fact that $\neg G r z \rightarrow \diamond \underline{n+1} \notin L_{n}$.

The $\mathcal{G}^{n}$ construction can be strengthened. Instead of duplicating the one point $n$, we can duplicate many points at once, defining a frame $\mathcal{G}_{S}$ by inserting a suitable new point $n^{\prime}$ in $\mathcal{G}$ between $n+1$ and $n$ and satisfying $\underline{n}$, for each $n \notin S$. This allows falsification of $A_{n}$ for all $n \notin S$ while preserving validity of $A_{n}$ when $n \in S$. Therefore

$$
\mathcal{G}_{S} \models A_{n} \quad \text { iff } \quad n \in S .
$$

There is a natural bounded morphism $f_{S}$ from $\mathcal{G}_{S}$ onto $\mathcal{G}$ : put $f_{S}\left(n^{\prime}\right)=n$ for all $n \notin S$, and otherwise $f_{S}(x)=x$. The existence of $f_{S}$ implies that the logic $L\left(\mathcal{G}_{S}\right)$ determined by $\mathcal{G}_{S}$ is included in $L(\mathcal{G})$, and so does not contain Grz. It follows that the $L\left(\mathcal{G}_{S}\right)$ 's form another family of $2^{\aleph_{0}}$ normal extensions of S4. $3_{\kappa}$ that lack the finite model property.

Now consider the (quasi-normal) logic $L\left(\mathcal{G}_{S}, \alpha\right)$, consisting of all formulas that are valid at $\alpha$ in $\mathcal{G}_{S}$. We have $\operatorname{Gr} z \notin L\left(\mathcal{G}_{S}, \alpha\right)$, and since $\alpha \models \neg \kappa$ but $\alpha \not \forall \square \neg \kappa$ in $\mathcal{G}_{S}, L\left(\mathcal{G}_{S}, \alpha\right)$ is not a normal logic. Moreover, since $\alpha R n$ for all $n$ we get that

$$
\mathcal{G}_{S}, \alpha \models A_{n} \quad \text { iff } \quad n \in S .
$$

Thus the $L\left(\mathcal{G}_{S}, \alpha\right)$ 's are a family of $2^{\aleph_{0}}$ non-normal extensions of $\mathrm{S} 4.3_{\kappa}$ that lack the finite model property.

Finally we note that if $\mathcal{G}_{S}^{-}$is the frame obtained by deleting $\beta$ from $\mathcal{G}_{S}$, then $\mathcal{G}_{S}^{-}$validates $G r z$. The $\operatorname{logics} L\left(\mathcal{G}_{S}^{-}\right)$are a family of $2^{\aleph_{0}}$ normal extensions of 
S4.3Gr $z_{\kappa}$, and the $\operatorname{logics} L\left(\mathcal{G}_{S}^{-}, \alpha\right)$ are a family of $2^{\aleph_{0}}$ non-normal extensions of $\mathrm{S} 4.3 G r z_{\kappa}$.

\section{Conclusion}

We have given a positive answer to the open question from [9] as to whether there are uncountably many maximally normal logics in the language with the modality $\square$ and the constant $\kappa$. The examples we constructed turned out to be logics that are not validated by any Kripke frame, and more strongly are not validated by any non-trivial complete algebra. We then showed that in the presence of weak transitivity, the number of maximally normal logics is finite, exhibiting eight such extensions of $\mathrm{K} 4_{\kappa}^{w}$, five of $\mathrm{K} 4_{\kappa}$, and three of $\mathrm{S} 4_{\kappa}$.

In addition we gave some results indicating that the set of logics in the language with $\kappa$ exhibits properties more like that of the the set of bimodal logics than the set of monomodal ones. These properties include that there are (continuum many) quasi-modal Post complete logics that are not normal; and that the extensions of S4. $3_{\kappa}$ include continuum many normal ones, and continuum many non-normal ones, none of which have the finite model property, as well as normal logics that are not finitely axiomatizable and ones that are undecidable.

A reviewer has noted that, apart from in the work on extensions of $\mathrm{S} 4.3_{\kappa}$, the logics we introduced were defined by frames in which $\kappa$ is a nominal, i.e. is interpreted as a singleton set of worlds. So many of the results hold also for hybrid logic with a single nominal. One can ask whether the results about extensions of S4.3 $3_{\kappa}$ also hold for this hybrid logic.

Finally, it might be asked whether the presence of $\kappa$ allows the construction of extensions of S4.3 $\kappa_{\kappa}$ that are incomplete for Kripke semantics (which cannot happen in the monomodal case). Here the answer would appear to be negative. Fine [8] showed that Kripke completeness is possessed by any monomodal finite width logic, i.e. any normal monomodal extension of $\mathrm{K} 4$ that, for some $n$, includes an axiom expressing that there are at most $n$ incomparable points $R$-related to a given point. His arguments involve working with languages that have a finite number of atomic formulas. It seems that by treating $\kappa$ as an additional atomic formula, these arguments still go through to show that in

the presence of $\kappa$, every finite width logic, including every normal extension of $\mathrm{S} 4.3_{\kappa}$, is complete for Kripke semantics. We leave it to interested readers to assess that for themselves.

\section{References}

[1] Galym Akishev and Robert Goldblatt. Monadic bounded algebras. Studia Logica, 96(1):1-40, 2010.

[2] Alan Ross Anderson. A reduction of deontic logic to alethic modal logic. Mind (New Series), 67:100-103, January 1958. 
[3] Guram Bezhanishvili, Silvio Ghilardi, and Mamuka Jibladze. An algebraic approach to subframe logics. modal case. Notre Dame Journal of Formal Logic, 52(2):187-202, 2011.

[4] R. A. Bull. That all normal extensions of S4.3 have the finite model property. Zeitschrift für Mathematische Logik und Grundlagen der Mathematik, 12:341-344, 1966.

[5] Alexander Chagrov and Michael Zakharyaschev. Modal Logic. Oxford University Press, 1997.

[6] Leo Esakia. Intuitionistic logic and modality via topology. Annals of Pure and Applied Logic, 127:155-170, 2004.

[7] Kit Fine. The logics containing S4.3. Zeitschrift für Mathematische Logik und Grundlagen der Mathematik, 17:371-376, 1971.

[8] Kit Fine. Logics containing K4. Part I. The Journal of Symbolic Logic, 39(1):31-42, 1974.

[9] Rohan French. Denumerably many Post-complete normal modal logics with propositional constants. Notre Dame Journal of Formal Logic, to appear.

[10] Robert Goldblatt. An algebraic study of well-foundedness. Studia Logica, 44(4):423-437, 1985.

[11] Paul R. Halmos. Algebraic Logic. Chelsea, New York, 1962.

[12] David Harel. Dynamic logic. In D. Gabbay and F. Guenthner, editors, Handbook of Philosophical Logic, Volume II: Extensions of Classical Logic, pages 497-604. D. Reidel, 1984.

[13] Bjarni Jónsson and Alfred Tarski. Boolean algebras with operators, part I. American Journal of Mathematics, 73:891-939, 1951.

[14] Tomasz Kowalski. Varieties of tense algebras. Reports on Mathematical Logic, 32:53-95, 1998.

[15] Tomasz Kowalski and Marcus Kracht. Semisimple varieties of modal algebras. Studia Logica, 83:351-363, 2006.

[16] Saul A. Kripke. Semantical analysis of modal logic II. Non-normal modal propositional calculi. In J. W. Addison, L. Henkin, and A. Tarski, editors, The Theory of Models, pages 206-220. North-Holland, 1965.

[17] Tadeusz Litak. On notions of completeness weaker than Kripke completeness. In Renate Schmidt, Ian Pratt-Hartmann, Mark Reynolds, and Heinrich Wansing, editors, Advances in Modal Logic, Volume 5, pages 149-169. King's College Publications, London, 2005. www . aiml .net. 
[18] D. C. Makinson. Some embedding theorems for modal logic. Notre Dame Journal of Formal Logic, 12:252-254, 1971.

[19] P. H. Nowell-Smith and E. J. Lemmon. Escapism: The logical basis of ethics. Mind (New Series), 69:289-300, July 1960.

[20] Emil L. Post. Introduction to a general theory of elementary propositions. American Journal of Mathematics, 43:163-185, 1921. Reprinted in [27], 264-283.

[21] Arthur N. Prior. Escapism: the logical basis of ethics. In A. I. Melden, editor, Essays in Moral Philosophy, pages 135-146. University of Washington Press, 1985.

[22] Krister Segerberg. An Essay in Classical Modal Logic, volume 13 of Filosofiska Studier. Uppsala Universitet, 1971.

[23] Krister Segerberg. Post completeness in modal logic. The Journal of Symbolic Logic, 37(4):711-715, 1972.

[24] Krister Segerberg. That every extension of S4.3 is normal. In Stig Kanger, editor, Proceedings of the Third Scandinavian Logic Symposium, pages 194196. North-Holland, 1975.

[25] Robert S. Streett. Propositional dynamic logic of looping and converse is elementarily decidable. Information and Control, 54:121-141, 1982.

[26] S. K. Thomason. Semantic analysis of tense logic. The Journal of Symbolic Logic, 37:150-158, 1972.

[27] Jean van Heijenoort, editor. From Frege to Gödel: a Source Book in Mathematical Logic, 1879-1931. Harvard University Press, 1967.

[28] Timothy Williamson. Continuum many maximal consistent normal bimodal logics with inverses. Notre Dame Journal of Formal Logic, 39(1):128134, 1998. 